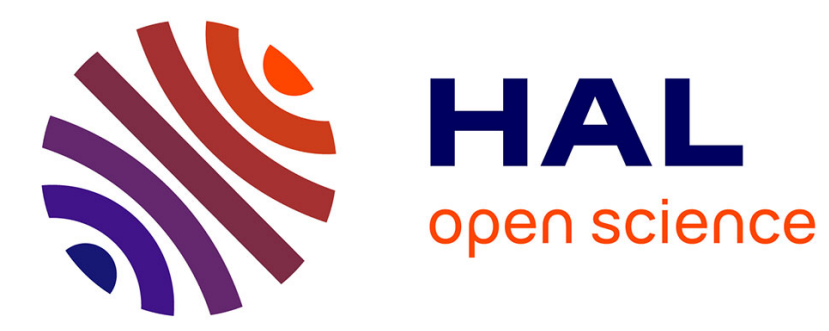

\title{
Persistence of abnormal cortisol levels in elderly persons after recovery from major depression.
}

Isabelle Beluche, Isabelle Chaudieu, Joanna Norton, Isabelle Carrière, Jean-Philippe Boulenger, Karen A. Ritchie, Marie-Laure Ancelin

\section{To cite this version:}

Isabelle Beluche, Isabelle Chaudieu, Joanna Norton, Isabelle Carrière, Jean-Philippe Boulenger, et al.. Persistence of abnormal cortisol levels in elderly persons after recovery from major depression.. Journal of Psychiatric Research, 2009, 43 (8), pp.777-83. 10.1016/j.jpsychires.2008.10.011 . inserm-00360626

\section{HAL Id: inserm-00360626 https://www.hal.inserm.fr/inserm-00360626}

Submitted on 11 Feb 2009

HAL is a multi-disciplinary open access archive for the deposit and dissemination of scientific research documents, whether they are published or not. The documents may come from teaching and research institutions in France or abroad, or from public or private research centers.
L'archive ouverte pluridisciplinaire HAL, est destinée au dépôt et à la diffusion de documents scientifiques de niveau recherche, publiés ou non, émanant des établissements d'enseignement et de recherche français ou étrangers, des laboratoires publics ou privés. 
Word count: 3708 words

2 Tables and 2 Figures

Persistence of abnormal cortisol levels in elderly persons after recovery from major depression

Isabelle Beluche $^{\mathrm{a}, 1}$, Ph.D., Isabelle Chaudieu ${ }^{\mathrm{a}, 1}, \mathrm{Ph} . \mathrm{D}$., Joanna Norton ${ }^{\mathrm{a}}$, MSc., Isabelle Carrière ${ }^{\mathrm{a}}$, Ph.D., Jean-Philippe Boulenger, ${ }^{\mathrm{a}, \mathrm{b}}$, M.D., Karen Ritchie ${ }^{\mathrm{a}}$, Ph.D., Marie L. Ancelin ${ }^{\mathrm{a}, 2}$, Ph.D.

${ }^{a}$ Inserm, U888, Montpellier, F-34093 France ; Univ Montpellier 1, Montpellier, F-34000 France ;

${ }^{\mathrm{b}} \mathrm{CHU}$ Montpellier, La Colombiere Hospital / University Department of Adult Psychiatry, Montpellier, F-34093 France.

${ }^{1}$ The two first authors contributed equally to this work

\section{${ }^{2}$ Corresponding author:}

Inserm U888, Hopital La Colombière, pav 42,

39 avenue Flahault, BP 34493, 34093 Montpellier Cedex 5, France

marie-laure.ancelin@inserm.fr

Tel: 334996145 62; Fax: 33499614579 


\section{Abstract}

Background: Cortisol hypersecretion is characteristic of acute clinical depression, but little is known in fully recovered, non-treated elderly persons with a life-time history of depression. This study was designed to examine patterns of diurnal cycle of cortisol in an elderly cohort without current depression or treatment for depression according to whether the person has or has not experienced a previous episode of depression or co-morbid depression with anxiety.

Methods: Cortisol secretion was evaluated in 162 community-dwelling elderly on a stressful and a non-stressful day (basal level). Past depression and anxiety disorders were assessed using a standardized psychiatric examination based on DSM-IV criteria (the Mini International Neuropsychiatric Interview).

Results: Antidepressant-free persons with a history of non-comorbid major depression $(6.8 \%$ of the sample) showed basal cortisol hypersecretion compared to those with depression and anxiety $(8.6 \%)$ or controls. Several hours after exposure to a stressful situation, controls showed a sustained increase in cortisol secretion, which was not observed in persons with a history of depression. Persons with a history of depression with anxiety showed a similar cortisol secretion at baseline to controls but a heightened response to stressful situation; a pattern comparable to that observed in subjects with pure anxiety disorders (16.7\%).

Conclusion: An abnormal HPA response persists even after effective treatment for depression. A history of co-morbid depression and anxiety gives rise to changes characteristic of anxiety alone. Our findings suggest that cortisol abnormalities may be trait markers for vulnerability to depression and for the differentiation of depression and depression with co-morbid anxiety.

Keywords: Anxiety co-morbidity; Cortisol; Depression; HPA axis; Stress; Trait. 


\section{Introduction}

One of the more consistent physiological abnormalities reported in major depression (MD) is cortisol hypersecretion, although studies in community-based samples have been less conclusive (see for review (Bhagwagar et al., 2008)). Cortisol hypersecretion was initially considered a state marker of MD, remitting with clinical improvement (see for reviews (Holsboer, 2000; Young, 2004)). More recently, however, persistent abnormalities have been noted in young non-depressed people at familial risk (Mannie et al., 2007) or in recovered depressed patients, particularly those at high risk of recurrence (Bhagwagar et al., 2003; Zobel et al., 2001), suggesting that such abnormalities could be markers of trait vulnerability to recurrent depression and may represent illness endophenotypes (Bhagwagar et al., 2008; Flint and Munafo, 2007; Hasler et al., 2004). This observation has not been investigated in the elderly, although hypothalamic-pituitary-adrenal (HPA) axis functioning may be especially vulnerable in this group given the accumulation of stressful events and high lifetime prevalence of MD (Ritchie et al., 2004). The study of elderly cohorts permits the examination of lifetime exposure to depression as well as patterns of recurrence.

Although some anxiety disorders also have been reported to be associated with neurobiological abnormalities, neither the lifetime persistence of cortisol abnormalities nor the impact of co-occurrence of depression with anxiety have been evaluated. Co-morbidity is common in the elderly (Lenze et al., 2000; Ritchie et al., 2004) and often indicative of more severe depressive symptoms, worse clinical course, and higher risk of suicide (Brown et al., 1996; Clayton, 1990; Lenze et al., 2000). The few studies which have been conducted to date on anxiety co-morbidity and cortisol secretion have focused on small clinical samples of young adults with current MD and post traumatic stress disorder (PTSD) or panic disorder, which are relatively uncommon in the elderly, in contrast with generalized anxiety disorder (GAD) or phobia (Ritchie et al., 2004). We recently reported an increased cortisol secretion in response to a naturalistic 
stressful situation in elderly people with GAD or phobia (Chaudieu et al., 2008). However, the neuroendocrine functioning in depression and the influence of co-morbidity in this elderly general population were not investigated.

The present study thus aims to evaluate if the diurnal cycle of salivary cortisol is modified in community-dwelling elderly persons who have recovered from a previous major depressive episode and whether co-morbidity with anxiety disorders could influence this pattern. Cortisol patterns were evaluated under two naturalistic but contrasting conditions, i.e. at home and following a stressful morning (hospital examinations).

\section{Methods}

\subsection{Study population}

The subjects (between 65 and 90 years) included in the analyses were part of the ESPRIT Project and selected by random sampling from the electoral rolls of the Montpellier district, in the South of France (Ritchie et al., 2004). The present analyses were conducted on a subsample of 201 subjects for whom salivary cortisol had been collected under both conditions, who were free of dementia and not being treated with medication likely to modify cortisol levels (glucocorticoids, hormonal replacement therapy, and benzodiazepines) (Chaudieu et al., 2008). From this sample, 18 subjects (eight women and ten men) reporting trauma exposure with distressing intrusions were further excluded, since we have previously reported that they have a modified pattern of basal cortisol level (Chaudieu et al., 2008). We also excluded subjects currently taking antidepressants (three women and five men) and two women with current MD, as well as 11 subjects with lifetime MD and co-morbid psychotic disorders or hypomania. This left 162 subjects in the present study. Written informed consent was obtained from all subjects, and the study was approved by the regional ethics committee. 


\subsection{Diagnostic instruments}

A standardized psychiatric examination validated within the French general population (Lecrubier et al., 1997); the Mini International Neuropsychiatric Interview MINI (French version 5.00), was used to detect lifetime MD or anxiety disorders according to DSM-IV criteria (American Psychiatric Association, 1994). Cases detected by the MINI were reviewed by a panel of psychiatrists to validate the initial diagnosis. The MINI was also used to detect subjects reporting previous severe trauma and meeting criteria A and B for DSM-IV post-traumatic stress disorder (PTSD). Current depressive symptomatology was evaluated using the Center for Epidemiologic Studies-Depression (CES-D) self-report questionnaire, the total score ranging from 0 to 60 . A cut-off point of 23 or more has been demonstrated to differentiate major depressive disorder (Radloff and Locke, 1986). Current use of antidepressants was validated either by presentation of the prescription or the medication itself, and the type of medication was recorded according to the World Health Organisation's ATC classification (W.H.O., 2000).

\subsection{Cortisol measurement}

Subjects were instructed not to drink, eat or smoke for at least $30 \mathrm{mn}$ before saliva collection. As cortisol levels increase shortly after awakening (Van Cauter et al., 1996), subjects were asked to start the protocol at least $1 \mathrm{~h}$ after awakening (mean time $1.5+0.8 \mathrm{~h}$ ) and subsequently twice with a 6-7h interval (the last sampling being collected before midnight) recording the exact time. Mean values for sampling time were $8.8+0.3,15.7+0.7$ and $21.7+0.6 \mathrm{~h}$. Cortisol levels were determined from saliva collection (Hellhammer et al., 1987) by direct radioimmunoassay (Diagnostic Systems Laboratories-Webster, Texas). Intra-assay and inter-assay coefficients of variation averaged $5 \%$. For less than $5 \%$ of the samples, the values were lower than the detection limit, $10 \mathrm{ng} / \mathrm{dl}(0.27 \mathrm{nM})$ and were replaced by a value corresponding to the threshold value divided by two. 


\subsection{Baseline and environmental stress conditions (home and hospital visit)}

As in other naturalistic studies, subjects were allowed to decide wake up and sleep times. Participants were encouraged to carry out their normal daily activities with limited physical exertion in order to maximize ecological validity. Samples were taken under two contrasting conditions; at the hospital ("stressful situation") where a lengthy clinical examination (between 08-11h AM) was undertaken involving various recognized psychosocial stressors (e.g. psychiatric examination, cognitive testing, clinical evaluation and blood collection) and a subsequent quiet day at home (baseline condition). The first sampling at the hospital was performed just before the clinical examination.

\subsection{Statistical analysis}

Since the distribution of raw cortisol is typically skewed, and the normal diurnal profile may be approximated by an exponential curve, raw values were log-transformed ${ }^{1}$. Given the non-fixed time sampling protocol, cortisol levels were calculated at fixed times $(8,15$, and $22 \mathrm{~h})$ from the regression of the three-cortisol values on the sampling times, for each subject and on two different days (basal and stressful situation). Area under the curves (AUC) were also standardized and calculated between $08 \mathrm{~h}$ and $22 \mathrm{~h}$ for each subject (extrapolating values from the equation of the regression line) as described previously (Chaudieu et al., 2008). Group comparisons were carried out using Student's t-test and analysis of variance for categorical explanatory variables. Individual stress responses, comparing home and hospital cortisol values, were performed using paired ttests. $p$ values $<0.05$ were considered to be statistically significant. Data were analyzed using SAS version 9.1 (Cary, NC).

\footnotetext{
${ }^{1}$ An absolute difference $(\delta)$ between two $\mathrm{LnC}$ values thus corresponds to a $\left(\mathrm{e}^{\delta}-1\right)$ x100 variation (expressed as \%) on initial (non log-transformed) cortisol concentrations.
} 


\section{Results}

The socio-demographic and clinical characteristics of the 162 subjects are given in Table 1 . The mean (SD) age was 72.8 (4.4). There was no effect of age (either as a continuous or categorical variable in three classes $(65-69 / 70-75 />75)$ on cortisol parameters. The participants selected for this study had no current MD and were not taking antidepressants. The level of current depressive symptomatology was low as attested by the CESD-score. The lifetime prevalence of psychiatric disorders was relatively high in this elderly population, $15.4 \%$ of persons having reported MD and 25.3\% anxiety disorder. Among the subjects with a history of MD, $44 \%$ reported two or three episodes. Median age of onset of MD was 49.5 years and the last depressive episode occurred several years before the cortisol sampling (median time 19.5 years).

Among the 25 subjects with a history of MD, 11 did not have co-morbid anxiety (the DEP group) and 14 did (the DEP/ANX group) (Table 2). Twenty-seven subjects reported lifetime anxiety disorders without co-morbid depression (ANX group). The remaining 110 subjects, with no history of psychiatric disorder or anti-depressant treatment constituted the control (CTL) group. The four groups did not differ significantly with regard to the non-psychiatric characteristics reported in Table 1, except for a significantly lower proportion of females in the CTL group (35.5\%) compared to the psychiatric groups $(48.1 \%$ in ANX, $72.7 \%$ in DEP, and $85.7 \%$ in DEP/ANX) $(p=0.0007)$ (data not shown). Regarding all the depression characteristics, there was also no significant difference between DEP and DEP/ANX groups.

Regarding paired (i.e. within group) comparisons, the CTL group, showed a significant increase in cortisol concentration between basal and stressful situation at $8 \mathrm{~h}, 15 \mathrm{~h}$ and $22 \mathrm{~h}$ (by $5.7 \%, 6.5 \%$, and $13.8 \%, \mathrm{p}<0.0001)$. DEP/ANX subjects showed a similar pattern of cortisol increase -although of higher amplitude- at $8 \mathrm{~h}$ (by 6.5\%, $\mathrm{p}=0.09$ ), $15 \mathrm{~h}$ (by $11.7 \%, \mathrm{p}=0.005$ ) and 
22h (by $27.7 \%, p=0.03$ ). For both CTL and DEP/ANX groups a significant increase in AUC was also observed (by $6.5 \%, p<0.0001$ and by $11.7 \%, p=0.005$, respectively) (data not shown). The profile of the DEP/ANX group was very close to that of the ANX subjects. On the other hand, DEP subjects, showed no significant increase in individual cortisol concentrations at 8,15 , or $22 \mathrm{~h}$ in paired comparisons between baseline and stressful situation.

A comparison of the four groups under baseline conditions, showed a nearly significant difference for morning cortisol ( $\mathrm{p}$ trend $=0.056$ ). Inter-group comparisons indicated a higher basal cortisol concentration at $8 \mathrm{~h}$ in the DEP group compared to both the DEP/ANX (by $10.8 \%$, $\mathrm{p}=0.02$ ), and CTL group (by $6.5 \%, \mathrm{p}=0.08$ ). The DEP/ANX and the ANX groups did not differ significantly from CTL. Under stressful conditions, the morning difference between the DEP and CTL groups was no longer significant due to a cortisol increase in the CTL but not the DEP group (as also observed in paired comparisons). A significant difference was observed at $15 \mathrm{~h}$ ( $\mathrm{p}$ trend $=0.014$ ) with a higher cortisol level in the DEP/ANX group compared to CTL (by $5.2 \%$, $\mathrm{p}=0.003$ ), as also observed for the ANX group (by $7.7 \%, \mathrm{p}=0.003$ ). The same pattern was observed at $22 \mathrm{~h}$ ( $\mathrm{p}$ trend $=0.008$ ) with a higher cortisol level in the DEP/ANX group compared to CTL (by 19.6\%, $\mathrm{p}=0.002$ ), as well as in the ANX group (by $16.2 \%, p=0.001$ ). Hence, the profiles of the DEP/ANX and ANX groups were comparable for both paired comparisons and group comparisons under basal and stressful situations.

Examining women and men separately, a significant difference was observed for morning cortisol under baseline conditions in women ( $p$ trend $=0.035$ ) but not in men, but the number of men $(n=5)$ is too low for adequate analysis (data not shown). In women, inter-group comparisons indicated a significantly higher basal cortisol concentration at $8 \mathrm{~h}$ in the DEP group ( $\mathrm{n}=8$ ) compared to the CTL ( $\mathrm{n}=39$, by $9.5 \%, \mathrm{p}=0.03)$ and DEP/ANX group $(\mathrm{n}=12$, by $14.0 \%, \mathrm{p}=0.002)$, this latter difference being also observed at $15 \mathrm{~h}(\mathrm{p}=0.04)$. The DEP/ANX group differed from 
controls, having higher cortisol levels following stressful situation at $22 \mathrm{~h}$ (by $16.2 \%, \mathrm{p}=0.03$ ). For DEP women, there was no significant increase in individual cortisol concentrations in paired comparisons, in contrast with the CTL and DEP/ANX or ANX groups, the two latter groups showing the highest values of relative differences (Figure 1).

Cortisol levels were further examined as a function of the recurrence of depressive episodes in DEP subjects. In contrast with the CTL group, there was no significant increase in relative differences between baseline and after exposure to stress, in paired comparisons at 8,15 , or $22 \mathrm{~h}$, both in persons reporting only one previous depressive episode $(n=7)$ and those with recurrent depression $(\mathrm{n}=4)$ (Figure 2). Compared to controls, morning basal cortisol was higher although non-significantly (by $3.9 \%$ ) in persons having reported only one past depressive episode, and the increase was $10.7 \%(\mathrm{p}=0.06)$ in those with recurrent past episodes (data not shown).

\section{Discussion}

This study explored for the first time the influence of past MD on diurnal cortisol variation in community-dwelling elderly subjects under naturalistic conditions. Our data indicate that in everyday functioning, daytime cortisol secretion in elderly subjects is modulated by previous history of depression. Faced with a stressful situation, elderly controls with no history of psychiatric disorder showed a sustained increase in cortisol secretion, several hours after exposure to a stressful situation. No such increase was observed in elderly persons reporting a previous episode of MD suggesting a persistent blunting of cortisol reactivity even after remission of depressive symptoms. On the other hand, persons with past depression and co-morbid anxiety have an even greater increase in HPA axis response than controls, a pattern comparable to that of 
subjects with past anxiety disorders without depression. Our data indicate that abnormal HPA reactivity in elderly subjects persist even after recovery from depression, with different cortisol patterns according to whether anxiety is also present.

\subsection{Methodological considerations and study design}

This naturalistic study examined the effect of exposure to a common stressful situation in the morning (which included various recognized psychosocial stressors) on subsequent diurnal cortisol levels at home. Our findings are thus more directly comparable to "post-event processing" research, i.e. the prolonged cognitive processing period following psychosocial interaction (Fehm et al., 2007), rather than to "recovery period" studies conducted shortly (1-2h) after exposure to a psychosocial stress test (Kudielka et al., 2004; Seeman et al., 1995).

In recovery period studies, it is generally assumed that cortisol levels return to normal about $2 \mathrm{~h}$ after the stressful situation, at least under laboratory conditions where specific stressors are tested. In our study, elderly subjects without psychiatric disorder showed enhanced cortisol levels from $4 \mathrm{~h}$ to $11 \mathrm{~h}$ after the stressful situation. To our knowledge, no other study has investigated long-term activity of the HPA axis following stress, notably during the "post-event processing" period. However, delayed recovery and sustained recurrent elevations in physiological autonomic nervous system response to emotional tasks have been observed in healthy adults, partly related to

rumination (Glynn et al., 2002). Rumination has been reported to last several days in young healthy adults following exposure to social situations (Fehm et al., 2007). This behavior may be an intervening variable, possibly exacerbated in the elderly.

\subsection{Cortisol response in depression without anxiety}

In our elderly population, persons reporting a history of MD without anxiety co-morbidity did not show significant increases in cortisol levels after exposure to stressful situation compared 
to controls. This appears to be due to high basal morning levels of cortisol, which could not be further increased after stress exposure, and suggests a persistent basal over-activation of the HPA axis. Morning basal hypersecretion of cortisol appeared to be increased in persons with recurrent depressive episodes. We do not know whether there is a gender difference in susceptibility to environmental conditions. While we observed a significant morning cortisol hypersecretion to characterize women with past depression compared to both controls and women with past depression plus anxiety, we could not confirm this pattern in men due to small numbers.

Previous research on basal cortisol hypersecretion has almost exclusively been conducted on in-patients with current MD, and rarely in patients with mild to moderate depression or outpatients (Bhagwagar et al., 2005; Copolov et al., 1989; Halbreich et al., 1985; Maes et al., 1994; Nelson and Davis, 1997; Stokes et al., 1984; Strickland et al., 2002). Our findings suggest that community-dwelling elderly with a history of MD, not taking antidepressants and currently free of depressive symptomatology, nonetheless continue to have abnormal cortisol pattern.

An absence of activation in response to stress in MD has been reported during the recovery period ("blunted" cortisol response), in laboratory stress studies with small samples, of predominantly young adults with current depression (Burke et al., 2005; Gotthardt et al., 1995; Trestman et al., 1991; Young et al., 2000b). The one previous study evaluating cortisol response in a naturalistic setting also observed a blunted HPA response to negative (but not positive) life events in MD in young depressed adults (Peeters et al., 2003). Our study extends these results to the period of "post-event processing" in community-dwelling elderly subjects and in (stressful or basal) naturalistic conditions, while taking into account for the first time past depressive episodes.

\subsection{Depression with anxiety}

Lifetime co-morbidity of depression with anxiety disorders is frequent in the elderly (Lenze et al., 2000; Ritchie et al., 2004). In our sample, it occurs in 56\% of subjects with MD, the most 
common forms being GAD and phobia (over $90 \%$ of anxiety disorders). Our results indicate that in persons with a previous history of depression with co-morbid anxiety, exposure to morning stress gives rise to increased evening cortisol levels, thus indicating a slowing in long-term recovery or, more likely, sustained and recurring cortisol elevations in the "post event processing" period. This pattern was comparable to that observed for subjects with anxiety disorders without co-morbid depression. Our results support Young et al.'s observation (Young et al., 2004) of an increased activation of the HPA axis, which persisted 75 minutes after the exposure to a psychosocial stressor in currently depressed patients with depression and anxiety compared to controls or subjects with depression only.

Examining the few studies, which have addressed the impact of depression with anxiety disorders on cortisol secretion, it appears that the neuroendocrine profile of currently depressed subjects actually depends on the nature of the associated anxiety disorder. Depressed subjects with co-morbid PTSD have a "PTSD-like" pattern, i.e. comparable to that of subjects with pure PTSD (Yehuda et al., 2004). Subjects with co-morbid panic disorder could also be considered as having a "panic disorder-like" pattern (also similar to that of depression) (Avery et al., 1985; Young et al., 2004). Our results suggest the existence of a comparable pattern in depressed subjects as a function of GAD or phobia co-morbidity, when dealing with the "post event processing period". Our observations could thus have nosological implications. Anxiety with depression is generally considered by clinicians to be a variant of a depressive disorder, however, these findings suggest that it may be a variant of an anxiety syndrome.

Past psychiatric disorders as well as anxiety co-morbidity have rarely been taken into consideration in cortisol studies in depressed subjects, which could explain some inconsistent results, depression having been associated with increased, decreased, or normal cortisol levels (Deuschle et al., 1998; Maes et al., 1994; Oldehinkel et al., 2001; Peeters et al., 2004; Strickland et al., 2002; Young et al., 2000a). This also highlights the important underlying problem of 
determining appropriate control groups for studies of neuroendocrine correlates of psychiatric disorder. Our results suggest that lifetime history and co-morbidity must be taken into consideration as they may modulate response even in currently normal subjects.

\subsection{Limitations and strength}

Although this population study had to be limited to 3-salivary measures, the basal characteristics of cortisol secretion are similar to previous studies with more frequent sampling (Ice et al., 2004). The systematic return of saliva samples by all subjects (including those with psychiatric disorders) and the choice of a non-fixed time sampling protocol (known to improve compliance particularly in the elderly (Jacobs et al., 2005; Kraemer et al., 2006)) within the loglinear zone far from awakening time, suggest our findings to be representative of naturalistic conditions. Although the prevalence of past psychiatric disorder is relatively high in our sample, our conclusions require further validation in other populations, in particular with regard to the question of whether our findings may be sex-specific. In our study design, we aimed to examine differences between baseline and naturalistic exposure to a stressful situation rather than to single experimental stressor. Randomization of days not being feasible, we commenced with stress exposure, thus obtaining maximal contrasting conditions (avoiding novelty or anticipatory effects

on baseline measures). Naturalistic conditions were performed the day after at home. Subjects did not report any additional stressors on the days they performed sampling. This raises however, the question as to whether the reduced reactivity and prolonged activation of the HPA system after stressful events may partly account for the increased cortisol secretion in baseline condition in the DEP group at $8 \mathrm{~h}$. It should be mentioned that cortisol levels in the DEP group were not significantly different from controls the first day at the hospital (even at 22h, more than $11 \mathrm{~h}$ after the stressful situation), whereas they only differed at home the day after at $8 \mathrm{~h}$ in the morning but not later at 15 or $22 \mathrm{~h}$. This thus makes unlikely that the higher morning cortisol level observed in 
the DEP group under baseline conditions could be due to prolonged activation of the HPA system after stressful events. Finally, we also controlled for eating, drinking, smoking and physical exertion.

\subsection{Conclusions}

Elderly persons with a previous history of anxiety disorder (with or without co-morbid depression) have abnormally elevated cortisol reactivity several hours after exposure to stress, whereas no such increase was observed in persons with a history of MD only. A past episode of MD on the other hand appears to give rise to a persistent increase in basal cortisol levels in the absence of current symptoms, which are not further elevated following exposure to stress. An episode of MD thus appears to be associated with long-term basal HPA hyperactivity through a largely unknown mechanism, with an apparent ceiling effect. While abnormal HPA axis functioning has been reported in relation to both anxiety and depression, our observations suggest that MD may give rise to a chronic impairment in cortisol reactivity even after the disappearance of symptoms and in the absence of treatment.

At a clinical level our observations suggest that cortisol readings may constitute a useful biological indicator in screening for anxiety co-morbidity, especially regarding the dramatic need for active identification of anxiety symptoms in late-life depression (Andreescu et al., 2007). The question is also raised as to whether endocrinological intervention may be desirable even after effective anti-depressant treatment in order to reduce the risk of subsequent episodes. Cortisol abnormalities have been characterized as long-term individual "trait" characteristics (Holsboer, 2000; Mannie et al., 2007; Young et al., 2000a). Cortisol levels could constitute markers of trait vulnerability to recurrent depression or illness endophenotypes (Bhagwagar et al., 2008; Flint and Munafo, 2007; Hasler et al., 2004). The question remains as to whether this is a direct consequence of depression or a reflection of an underlying vulnerability. Follow-up studies with 
this cohort will further address this question and examine the temporal relationship between changes in HPA axis functioning and psychiatric disorder. 


\section{References}

American Psychiatric Association. Diagnostic and Statistical Manual of Mental Disorders (DSMIV). Washington, DC: American Psychiatric Press, 1994

Andreescu C, Lenze EJ, Dew MA, Begley AE, Mulsant BH, Dombrovski AY, et al. Effect of comorbid anxiety on treatment response and relapse risk in late-life depression: controlled study. Br J Psychiatry. 2007;190:344-9.

Avery DH, Osgood TB, Ishiki DM, Wilson LG, Kenny M, Dunner DL. The DST in psychiatric outpatients with generalized anxiety disorder, panic disorder, or primary affective disorder. Am J Psychiatry 1985;142:844-8.

Bhagwagar Z, Hafizi S, Cowen PJ. Increase in concentration of waking salivary cortisol in recovered patients with depression. Am J Psychiatry. 2003;160:1890-1.

Bhagwagar Z, Hafizi S, Cowen PJ. Increased salivary cortisol after waking in depression. Psychopharmacology (Berl). 2005;182:54-7.

Bhagwagar Z, Hafizi S, Cowen PJ. 'It's not over when it's over': persistent neurobiological abnormalities in recovered depressed patients. Psychol Med 2008;38:307-313.

Brown C, Schulberg HC, Madonia MJ, Shear MK, Houck PR. Treatment outcomes for primary care patients with major depression and lifetime anxiety disorders. Am J Psychiatry $1996 ; 153: 1293-300$.

Burke HM, Davis MC, Otte C, Mohr DC. Depression and cortisol responses to psychological stress: a meta-analysis. Psychoneuroendocrinology 2005;30:846-56.

Chaudieu I, Beluche I, Norton J, Boulenger JP, Ritchie K, Ancelin ML. Abnormal reactions to environmental stress in elderly persons with anxiety disorders: evidence from a population study of diurnal cortisol changes. J Affect Disord 2008; 106:307-313. 
Clayton PJ. The comorbidity factor: establishing the primary diagnosis in patients with mixed symptoms of anxiety and depression. J Clin Psychiatry 1990;51 Suppl:35-9.

Copolov DL, Rubin RT, Stuart GW, Poland RE, Mander AJ, Sashidharan SP, et al. Specificity of the salivary cortisol dexamethasone suppression test across psychiatric diagnoses. Biol Psychiatry 1989;25:879-93.

Deuschle M, Weber B, Colla M, Depner M, Heuser I. Effects of major depression, aging and gender upon calculated diurnal free plasma cortisol concentrations: a re-evaluation study. Stress $1998 ; 2: 281-7$

Fehm L, Schneider G, Hoyer J. Is post-event processing specific for social anxiety? J Behav Ther Exp Psychiatry 2007;38:11-22.

Flint J, Munafo MR. The endophenotype concept in psychiatric genetics. Psychol Med. $2007 ; 37: 163-80$.

Glynn LM, Christenfeld N, Gerin W. The role of rumination in recovery from reactivity: cardiovascular consequences of emotional states. Psychosom Med 2002;64:714-26.

Gotthardt U, Schweiger U, Fahrenberg J, Lauer CJ, Holsboer F, Heuser I. Cortisol, ACTH, and cardiovascular response to a cognitive challenge paradigm in aging and depression. Am J Physiol 1995;268:R865-73.

Halbreich U, Asnis GM, Shindledecker R, Zumoff B, Nathan RS. Cortisol secretion in endogenous depression. II. Time-related functions. Arch Gen Psychiatry 1985;42:909-14.

Hasler G, Drevets WC, Manji HK, Charney DS. Discovering endophenotypes for major depression. Neuropsychopharmacology. 2004;29:1765-81.

Hellhammer DH, Kirschbaum C, Belkien L. Measurement of salivary cortisol under psychological stimulation. In Hingtgen J.N., Hellhammer D.H., Huppman G. (Eds.), Adavanced Methods in Psychobiology Toronto: Hogrefe, 1987. (pp. 281-289). 
Holsboer F. The corticosteroid receptor hypothesis of depression. Neuropsychopharmacology $2000 ; 23: 477-501$

Ice GH, Katz-Stein A, Himes J, Kane RL. Diurnal cycles of salivary cortisol in older adults. Psychoneuroendocrinology 2004;29:355-70

Jacobs N, Nicolson NA, Derom C, Delespaul P, van Os J, Myin-Germeys I. Electronic monitoring of salivary cortisol sampling compliance in daily life. Life Sci 2005;76:2431-43.

Kraemer HC, Giese-Davis J, Yutsis M, O'Hara R, Neri E, Gallagher-Thompson D, et al. Design decisions to optimize reliability of daytime cortisol slopes in an older population. Am J Geriatr Psychiatry. 2006;14:325-33.

Kudielka BM, Buske-Kirschbaum A, Hellhammer DH, Kirschbaum C. HPA axis responses to laboratory psychosocial stress in healthy elderly adults, younger adults, and children: impact of age and gender. Psychoneuroendocrinology 2004;29:83-98.

Lecrubier Y, Sheehan D, Weiller E, Amorim P, Bonara I, Sheehan K, et al. The Mini International Neuropsychiatric Interview (MINI), a short diagnostic interview: reliability and validity according to the CIDI. European Psychiatry 1997;12:232-241.

Lenze EJ, Mulsant BH, Shear MK, Schulberg HC, Dew MA, Begley AE, et al. Comorbid anxiety disorders in depressed elderly patients. Am J Psychiatry. 2000;157:722-8.

Maes M, Calabrese J, Meltzer HY. The relevance of the in- versus outpatient status for studies on HPA-axis in depression: spontaneous hypercortisolism is a feature of major depressed inpatients and not of major depression per se. Prog Neuropsychopharmacol Biol Psychiatry $1994 ; 18: 503-17$.

Mannie ZN, Harmer CJ, Cowen PJ. Increased waking salivary cortisol levels in young people at familial risk of depression. Am J Psychiatry. 2007;164:617-21.

Nelson JC, Davis JM. DST studies in psychotic depression: a meta-analysis. Am J Psychiatry 1997;154:1497-503. 
Oldehinkel AJ, van den Berg MD, Flentge F, Bouhuys AL, ter Horst GJ, Ormel J. Urinary free cortisol excretion in elderly persons with minor and major depression. Psychiatry Res 2001;104:39-47.

Peeters F, Nicholson NA, Berkhof J. Cortisol responses to daily events in major depressive disorder. Psychosom Med 2003;65:836-41.

Peeters F, Nicolson NA, Berkhof J. Levels and variability of daily life cortisol secretion in major depression. Psychiatry Res 2004;126:1-13.

Radloff LS, Locke BZ. The community mental health assessment survey and CES-D scale. Community surveys of psychiatric disorders. New Brunswick, NJ: Rutgers University Press, 1986

Ritchie K, Artero S, Beluche I, Ancelin ML, Mann A, Dupuy AM, et al. Prevalence of DSM-IV psychiatric disorder in the French elderly population. Br J Psychiatry 2004;184:147-52.

Seeman TE, Singer B, Charpentier P. Gender differences in patterns of HPA axis response to challenge: Macarthur studies of successful aging. Psychoneuroendocrinology 1995;20:71125 .

Stokes PE, Stoll PM, Koslow SH, Maas JW, Davis JM, Swann AC, et al. Pretreatment DST and hypothalamic-pituitary-adrenocortical function in depressed patients and comparison groups. A multicenter study. Arch Gen Psychiatry 1984;41:257-67.

Strickland PL, Deakin JF, Percival C, Dixon J, Gater RA, Goldberg DP. Bio-social origins of depression in the community. Interactions between social adversity, cortisol and serotonin neurotransmission. Br J Psychiatry 2002;180:168-73.

Trestman RL, Coccaro EF, Bernstein D, Lawrence T, Gabriel SM, Horvath TB, et al. Cortisol responses to mental arithmetic in acute and remitted depression. Biol Psychiatry 1991;29:1051-4. 
Van Cauter E, Leproult R, Kupfer DJ. Effects of gender and age on the levels and circadian rhythmicity of plasma cortisol. J Clin Endocrinol Metab 1996;81:2468-73.

W.H.O. World Health Organising Collaborating Centre for Drug Statistics Methodology. Guidelines for ATC classification and DDD assignment. Oslo, Norway., 2000

Yehuda R, Halligan SL, Golier JA, Grossman R, Bierer LM. Effects of trauma exposure on the cortisol response to dexamethasone administration in PTSD and major depressive disorder. Psychoneuroendocrinology 2004;29:389-404.

Young AH. Cortisol in mood disorders. Stress. 2004;7:205-8.

Young EA, Abelson J, Cameron OG. Effect of comorbid anxiety disorders on the hypothalamicpituitary-adrenal axis response to a social stressor in major depression. Biol Psychiatry 2004;56:113-20.

Young EA, Aggen SH, Prescott CA, Kendler KS. Similarity in saliva cortisol measures in monozygotic twins and the influence of past major depression. Biol Psychiatry 2000a;48:704.

Young EA, Lopez JF, Murphy-Weinberg V, Watson SJ, Akil H. Hormonal evidence for altered responsiveness to social stress in major depression. Neuropsychopharmacology $2000 \mathrm{~b} ; 23: 411-8$

Zobel AW, Nickel T, Sonntag A, Uhr M, Holsboer F, Ising M. Cortisol response in the combined dexamethasone/CRH test as predictor of relapse in patients with remitted depression. a prospective study. J Psychiatr Res. 2001;35:83-94. 


\section{Figure Legend}

Figure 1. Relative differences in cortisol concentrations during diurnal secretion in women with or without past major depression and anxiety disorders

The figure shows the relative differences (mean + S.E.M.) for women without past psychiatric disorders (CTL), women reporting past MD without anxiety co-morbidity (DEP), women with past depression and anxiety co-morbidity (DEP/ANX), and women with past non co-morbid anxiety disorders (ANX). Relative differences are expressed as the differences between hospital and baseline conditions divided by the basal values.

Figure 2. Relative differences in cortisol concentrations during diurnal secretion as a function of the number of past major depressive episodes in persons without lifetime anxiety co-morbitiy.

The figure shows the relative differences (mean + S.E.M.) for persons reporting a past episode of MD without co-morbid anxiety (DEP group). Relative differences are expressed as the differences between hospital and baseline conditions divided by the basal values. History of depression was expressed as the number of past major depressive episodes (MDE), 0 for persons reporting no past psychiatric disorders (CTL), 1 for only one MDE, and $>2$ corresponds to those who have reported at least two MDE. 
Table 1

Socio-demographic and clinical characteristics of the study population

$(\mathrm{n}=162)$

Female (\%)

44.4

Mean age (SD)

$72.8(4.4)$

Low education level ${ }^{1}(\%)$

47.5

Current smoker (\%)

6.2

MMSE score (mean (SD))

Body Mass Index in $\mathrm{kg} / \mathrm{m}^{2}$ (mean (SD))

CESD score (mean (SD))

Lifetime psychiatric disorders:

Major Depression (\%)

Number of previous depressive episodes ${ }^{2}$

$$
1(\%)
$$

$>2(\%)$

44.0

Age of onset of major depression ${ }^{2}$ (median (min-max))

Time between the cortisol sampling and the last depressive episode ${ }^{2}$ (median (min-max))

$19.5(2.3-38.1)$

Phobia (\%)

Panic disorder $(\%)$

At least one anxiety disorder (\%)

Co-morbidity (DEP + ANX) $(\%)$

8.6

${ }^{1} 9$ years of schooling or less.

${ }^{2}$ For subjects with a history of major depression 
Table 2

Cortisol levels under baseline and stressful conditions according to the presence of history of major depression without or with co-morbid anxiety disorders

\begin{tabular}{|c|c|c|c|c|c|c|c|c|c|c|}
\hline \multirow{3}{*}{$\begin{array}{l}\text { Cortisol } \\
\text { parameters }\end{array}$} & \multirow{3}{*}{$\begin{array}{l}\text { Group }^{b} \\
\text { (CTL: } n=110 \text { ) } \\
\text { (DEP: } n=11 \text { ) } \\
\text { (DEP/ANX: } n=14 \text { ) } \\
\text { (ANX: } n=27 \text { ) }\end{array}$} & \multicolumn{8}{|c|}{ Group comparisons } & \multirow{3}{*}{$\begin{array}{l}\text { Paired } \\
\text { comparisons } \\
p\end{array}$} \\
\hline & & \multicolumn{3}{|c|}{ Baseline day } & \multicolumn{3}{|c|}{ Stressful day } & \multicolumn{2}{|c|}{$\begin{array}{l}\text { Relative difference } \\
(\Delta)^{\mathrm{c}}\end{array}$} & \\
\hline & & Mean & SD & $p^{\mathrm{d}}$ & Mean & $\mathrm{SD}$ & $p^{\mathrm{d}}$ & $\%$ & $p$ & \\
\hline \multirow{4}{*}{$\mathrm{LnC}_{8 \mathrm{~h}}{ }^{\mathrm{a}}$} & CTL & 5.41 & 0.63 & \multirow[t]{4}{*}{$0.056^{1}$} & 5.66 & 0.62 & \multirow[t]{4}{*}{0.46} & 5.7 & \multirow[t]{4}{*}{0.42} & $<0.0001$ \\
\hline & DEP & 5.76 & 0.59 & & 5.64 & 0.77 & & -1.6 & & 0.95 \\
\hline & DEP/ANX & 5.20 & 0.51 & & 5.49 & 0.57 & & 6.5 & & 0.09 \\
\hline & ANX & 5.64 & 0.72 & & 5.83 & 0.83 & & 4.5 & & 0.004 \\
\hline \multirow{4}{*}{$\mathrm{LnC}_{15 \mathrm{~h}}{ }^{\mathrm{a}}$} & CTL & 4.21 & 0.61 & \multirow[t]{4}{*}{0.14} & 4.44 & 0.47 & $0.014^{2}$ & 6.5 & \multirow[t]{4}{*}{0.28} & $<0.0001$ \\
\hline & DEP & 4.47 & 0.63 & & 4.52 & 0.46 & & 2.1 & & 0.43 \\
\hline & DEP/ANX & 4.23 & 0.53 & & 4.67 & 0.41 & & 11.7 & & 0.005 \\
\hline & ANX & 4.49 & 0.68 & & 4.78 & 0.70 & & 7.3 & & 0.17 \\
\hline \multirow{4}{*}{$\mathrm{LnC}_{22 \mathrm{~h}}{ }^{\mathrm{a}}$} & CTL & 3.02 & 0.96 & \multirow[t]{4}{*}{0.40} & 3.21 & 0.73 & $0.0008^{3}$ & 13.8 & \multirow[t]{4}{*}{0.52} & $<0.0001$ \\
\hline & DEP & 3.18 & 0.92 & & 3.39 & 0.71 & & 12.3 & & 0.24 \\
\hline & DEP/ANX & 3.27 & 0.83 & & 3.84 & 0.57 & & 27.7 & & 0.03 \\
\hline & ANX & 3.34 & 1.00 & & 3.73 & 0.82 & & 19.0 & & 0.004 \\
\hline
\end{tabular}

${ }^{\mathrm{a}}$ Time concentrations correspond to the Ln of cortisol concentration expressed as ng/dl

${ }^{\mathrm{b}}$ Subjects without lifetime psychiatric disorders (CTL), a history of depression (DEP) depression with anxiety (DEP/ANX) or anxiety (ANX).

${ }^{c}$ Corresponds to the mean of individual relative differences which is the mean of the ratio [(hospital - home)/ home] expressed as \%.

d -values for two-by-two inter-group comparisons are indicated between brackets (if not reported, $p>0.10$ ):

${ }^{1}$ [DEP vs. CTL: $\mathrm{p}=0.08$; DEP vs. DEP/ANX: $\left.\mathrm{p}=0.02\right]$

${ }^{2}[\mathrm{DEP} / \mathrm{ANX}$ vs. CTL, $\mathrm{p}=0.003 ;$ ANX vs. CTL, $\mathrm{p}=0.003]$

${ }^{3}[\mathrm{DEP} / \mathrm{ANX}$ vs. CTL: $\mathrm{p}=0.002$; ANX vs. CTL, $\mathrm{p}=0.001$; DEP vs. DEP/ANX: $\mathrm{p}=0.09]$ 


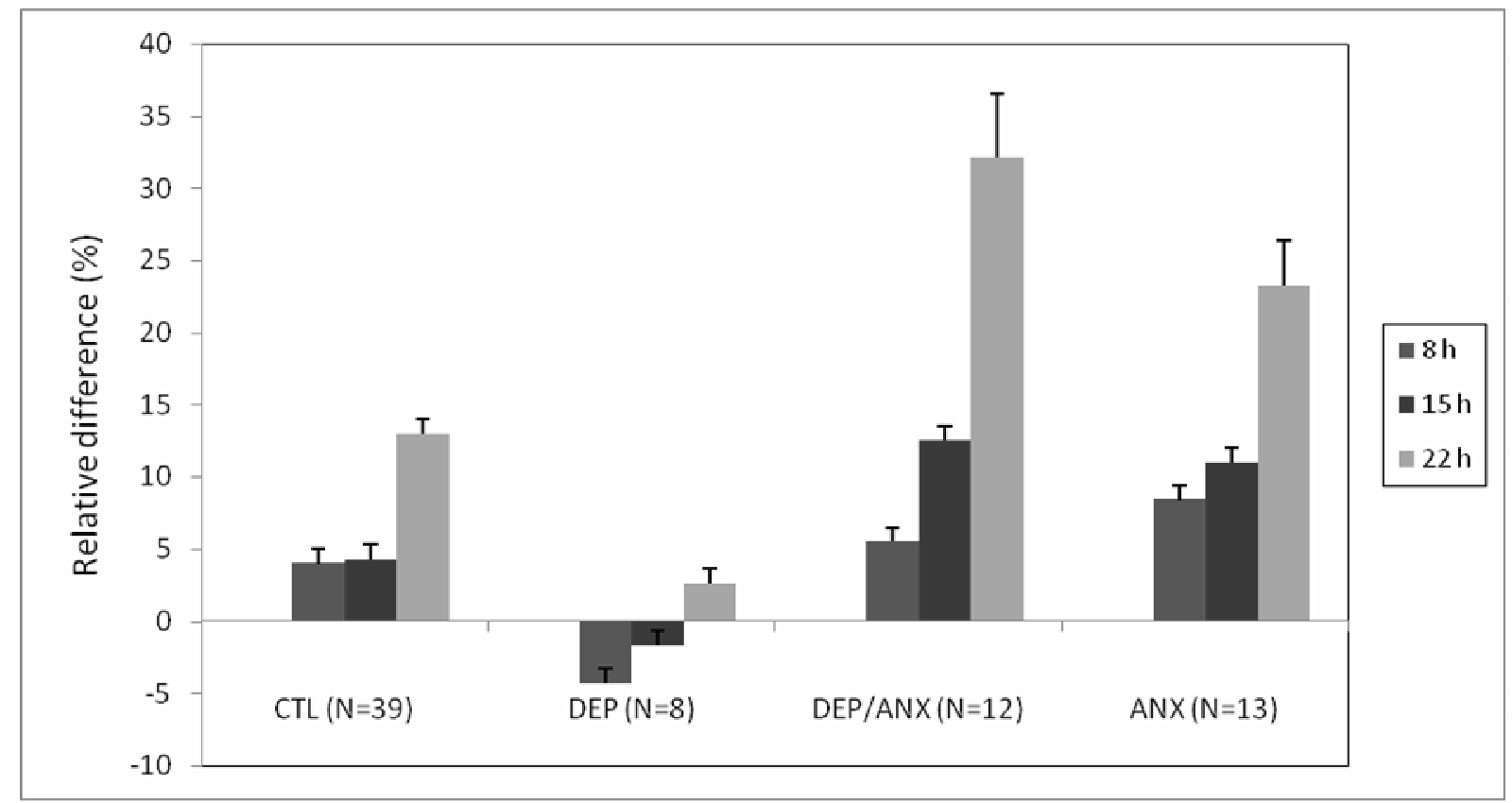

Figure 1 


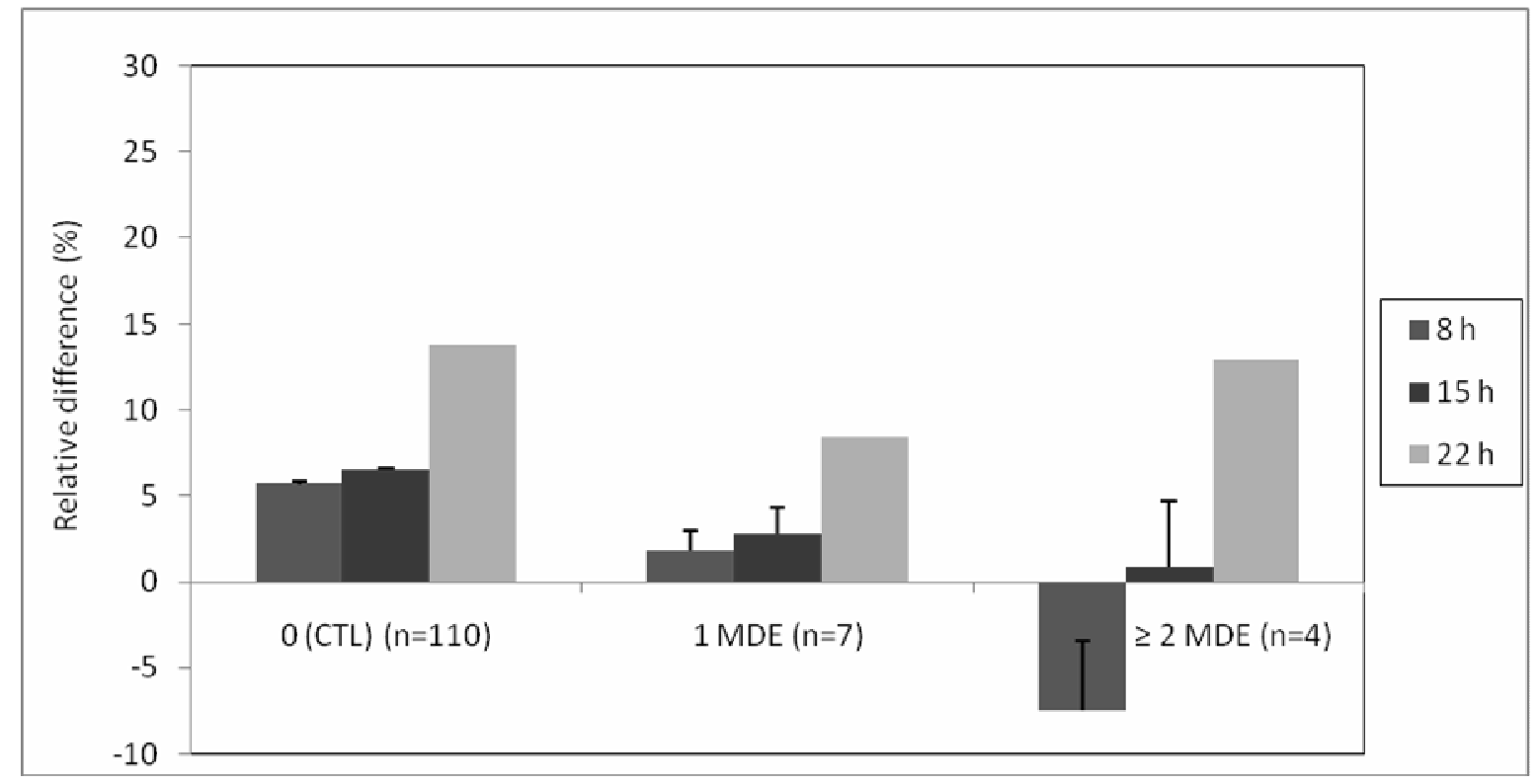

Figure 2 